\title{
The Value of Screening for Celiac Disease in Systemic Lupus Erythematosus: A Single Experience of a Tertiary Medical Center
}

\author{
Fahidah AlEnzi • Mada Yateem • Manal Shaikh • Fahad AlSohaibani • \\ Badryah Alhaymouni · AlShaikh Ahmed · Sulaiman M. Al-Mayouf
}

Received: June 6, 2020 / Published online: July 23, 2020

(C) The Author(s) 2020

\section{ABSTRACT}

Introduction: Systemic lupus erythematosus (SLE) is a multi-organ inflammatory disease associated with autoimmune diseases. The aim of the study is to assessed the frequency of celiac disease (CD) in adults and children with SLE (aSLE and cSLE, respectively) and compare them with rheumatoid arthritis (RA) and juvenile idiopathic arthritis (JIA) patients; the study also explored the clinical impact of CD serologic markers on SLE disease activity and severity.

Methods: This was a cross-sectional study. Patients with SLE who had regular follow-up in rheumatology clinics were evaluated for

Digital Features To view digital features for this article go to https://doi.org/10.6084/m9.figshare.12661592.

F. AlEnzi $(\bowtie)$

Clinical Sciences, College of Medicine, Princess Nourah Bint Abdulrahman University, Riyadh, Saudi Arabia

e-mail: Fmalenzi@pnu.edu.sa

B. Alhaymouni · A. Ahmed

Adult Rheumatology, King Faisal Specialist Hospital and Research Center, Riyadh, Saudi Arabia

M. Yateem · M. Shaikh · S. M. Al-Mayouf Pediatric Rheumatology, King Faisal Specialist

Hospital and Research Center, Riyadh, Saudi Arabia

F. AlSohaibani

Adult Gastroenterology, King Faisal Specialist

Hospital and Research Center, Riyadh, Saudi Arabia laboratory and clinical variables using serology and the SLE Disease Activity Index (SLEDAI). To assess the occurrence of CD serology in cSLE and aSLE and the clinical impact of CD serologic markers on SLE, patients were tested for antigliadin (AGA), anti-endomysium (EmA) and anti-tissue transglutaminase (tTG) antibodies. RA and JIA patients were included for comparison. Duodenal biopsy was conducted in patients who exhibited CD markers.

Results: The CD marker was found in 29 $(11.6 \%)$ of the 250 patients. AGA was present in seven aSLE patients and tTG in two (11.1\%). Among cSLE patients, the autoantibody was present in $17.6 \%$ (AGA in four, tTG in two, and EmA in three). For RA patients, five had AGA and tTG and one had EmA, with an overall positivity of $9.7 \%$. Five JIA patients had AGA (four with EmA and five with tTG) with overall positivity of $10.9 \%$; the serum IgA level was normal in all patients except one. Duodenal endoscopic biopsy was performed in patients with positive $\mathrm{CD}$ markers (two declined). Histologic confirmation of $\mathrm{CD}$ was reported in one RA and one JIA patient but in none of the SLE patients. There was no correlation between the presence of CD markers and autoantibodies in SLE.

Conclusion: CD antibodies did not influence SLE activity. Thus, SLE patients may not need to be screened for CD antibodies. 
Keywords: Celiac disease; Juvenile idiopathic arthritis; Rheumatoid arthritis; Systemic lupus erythematosus

\section{Key Summary Points}

Many autoimmune diseases have been described as occurring concomitantly with celiac disease (CD).

There is an increased frequency of positive serologic celiac disease markers in patients with systemic lupus erythematosus (SLE).

The presence of serologic celiac disease markers did not influence SLE disease activity.

\section{INTRODUCTION}

Systemic lupus erythematosus (SLE) is a systemic inflammatory disease characterized by the production of autoantibodies and multisystem involvement. Gastrointestinal manifestations are common among SLE patients because of the disease itself, as a consequence of infection, or because of side effects of medications. These manifest in a wide spectrum, including recurrent abdominal pain, vomiting, malabsorption, enteritis, pancreatitis, and others [1-3]. T-cell-induced immune-mediated disease triggered by gluten-containing food ingestion is known to be a cause of celiac disease (CD) among genetically predisposed individuals [4]. It has diverse clinical presentations, ranging from enteropathy with signs of severe malabsorption to asymptomatic presentation [5-7]. Among autoimmune diseases, CD is one of the most frequently diagnosed in children with increasing diagnosis rates among adult patients $[8,9]$. In recent times, the prevalence rate of $\mathrm{CD}$ has been reported be high among children in Saudi Arabia compared to that in Europe and North America [10].

In general, the probability of developing a second disorder is high among patients with autoimmune disorders. Many autoimmune diseases have been described as occurring concomitantly with CD. Several screening studies have shown an increased prevalence of $C D$ among individuals with autoimmune diseases, such as diabetes mellitus type I, juvenile idiopathic arthritis (JIA), autoimmune thyroiditis, and rheumatoid arthritis (RA) [11-13]. In a previous study, we showed that screening for asymptomatic CD in children with JIA might be useful [14].

The association of SLE with CD has been frequently reported. Genetic predisposition plays a vital role in establishing the association between SLE and CD but is yet to be fully understood. CD and SLE share histocompatibility antigens of specific leukocytes, which may indicate that these haplotypes carry a higher risk for developing both diseases [15]. The factual incidence of CD and its autoantibodies in SLE is still not well documented [16-18]. To the best of our knowledge, there is no published evidence relating to the coexistence of $\mathrm{CD}$ among SLE patients in Saudi Arabia. In this study, the frequency of CD in adults and children with SLE (aSLE and cSLE) was assessed and compared with the presence of RA and JIA. Furthermore, we investigated the clinical impact of CD on SLE disease activity.

\section{METHODS}

\section{Participant and Study Design}

In this cross-sectional study, patients with aSLE and CSLE who fulfilled the criteria of the Systemic Lupus International Collaborating Clinics (SLICC) and with a regular follow-up at King Faisal Specialist Hospital and Research Center, Riyadh (KFSHRC-R), at the rheumatology department were included [19]. Patients $>14$ years of age were moved to the adult rheumatology department for comprehensive care as per the hospital policy. Accordingly, our definition for cSLE was patients up to 14 years. As a comparison group, patients with RA and JIA were included $[20,21]$.

All patients were interviewed for their demographic data, disease manifestation, and laboratory results, which were collected to 
calculate the overall SLE disease activity using the Systemic Lupus Erythematosus Disease Activity Index (SLEDAI) and to determine the clinical form of the disease [22].

All patients had a complete blood count (CBC) with differential count, C-reactive protein (CRP), erythrocyte sedimentation rate (ESR), iron profile, renal and hepatic profile, serum IgA level, and serologic markers of CD including IgG and IgA antigliadin-antibodies (AGAG and AGAA), IgA anti-endomysium (EmA), and anti-tissue transglutaminase (tTG) antibodies using the indirect immunofluorescence and standard ELISA methods recorded. Additionally, laboratory tests, such as complement (C3 C4) and the anti-nuclear antibody (ANA) profile including the antiphospholipid antibody profile, anti-double-stranded DNA antibody, urine protein creatinine ratio, and urinalysis of all SLE patients, were assessed $[23,24]$. Patients with positive serologic markers of $\mathrm{CD}$ were interviewed and informed about the results and the need for a confirmatory upper endoscopy with duodenal-jejunal biopsy and histopathologic examination. In SLE patients with equivocal small-bowel histologic findings, HLA-DQ2 and -DQ8 tests were ordered to include the diagnosis of $\mathrm{CD}[25,26]$.

The study was approved by the Research Affairs Council at KFSHRC-R. All collected data were analyzed with due confidentiality practice, and no personal identity was needed. All procedures performed involving human participants were performed in accordance with the ethical standards of the institutional and/or national research committee and with the 1964 Helsinki Declaration and its later amendments or comparable ethical standards. Informed consent was obtained from all adult participants and from the parents of all enrolled children.

\section{Statistical Analysis}

Descriptive statistics for continuous variables were reported as mean \pm standard deviation (SD), while categorical variables were expressed as frequencies and percentages. Chi-square and Fisher's exact tests were used to compare qualitative variables. The Mann-Whitney test was performed to compare the two groups (SLE and arthritis patients). Statistical significance was defined as $P<0.05$.

\section{RESULTS}

The study group consisted of 250 patients meeting the eligibility criterion, selected at random. Among these, 81 patients had aSLE with 34.1 (11.5) years as mean age, 34 patients had cSLE with 10.3 (2.7) years as mean age, 62 patients had RA with 48.8 [10] years as mean age, and 72 patients had JIA with 10.0 (2.6) years as mean age. The age and disease duration did not exhibit any significant statistical differences between aSLE and RA patients and cSLE and JIA patients ( $P$ 0.18). However, there was a statistically significant gender difference among all groups; females were predominant $(P=0.03)$. Twenty-nine patients tested positive for serologic markers of $\mathrm{CD}$, and several patients had more than one positive $\mathrm{CD}$ marker. The rate of serologic $C D$ positivity was higher among SLE patients (13.1\%) than among arthritis (RA and JIA) patients (10.4\%). However, the difference was not statistically significant $(P=0.27)$. Nine $(11.1 \%)$ aSLE patients were positive for CD markers: four had AGAG, three had AGAA, and two had tTG. However, six (17.6\%) cSLE patients had positive CD markers; three had positive AGAG, one had AGAA, three had positive EmA, and two had tTG. In contrast, six $(9.7 \%)$ RA patients tested positive: four patients with AGAG, one with AGAA, and one patient with EmA and tTG; eight (10.9\%) tested positive: five with AGAG, four with EmA, and five with tTG. All patients had normal serum IgA levels, but one SLE patient had low serum IgA levels. Most patients with isolated positive AGAA and AGAG showed slightly elevated serum IgA levels. However, six patients with EmA and tTG had markedly elevated AGAA and AGAG levels. Antigliadin antibodies were found in $8.6 \%$ of aSLE and $11.8 \%$ of cSLE patients. In contrast, patients with cSLE and JIA had more EmA and tTG positivity than did the adult patients. However, these differences were statistically insignificant. Table 1 shows the 
Table 1 Demographic features and frequency and distribution of serologic markers of celiac disease

\begin{tabular}{llllll}
\hline & aSLE & cSLE & RA & JIA & P value \\
\hline Number & 81 & 34 & 62 & 73 & \\
Female/male & $76 / 5$ & $28 / 6$ & $58 / 4$ & $45 / 28$ & 0.03 \\
Age (mean \pm SD) years & $34.1 \pm 11.5$ & $10.3 \pm 2.7$ & $48.8 \pm 10$ & $10.0 \pm 2.6$ & 0.18 \\
Patients with positive CD markers* & $9(11 \%)$ & $6(17.6 \%)$ & $6(9.6 \%)$ & $8(10.9 \%)$ & 0.6 \\
AGAA & 3 & 1 & 1 & 0 & 0.3 \\
AGAG & 4 & 3 & 4 & 4 & 0.5 \\
EmA & 0 & 3 & 1 & 4 & 0.13 \\
tTG & 2 & 2 & 0 & 5 & 0.4 \\
\hline
\end{tabular}

aSLE adult systemic lupus erythematosus, $C S L E$ childhood systemic lupus erythematosus, $R$ : rheumatoid arthritis, $J I A$ juvenile idiopathic arthritis, $C D$ celiac disease, $A G A A$ IgA antigliadin antibodies, $A G A G$ IgG antigliadin antibodies, $E m A$ IgA anti-endomysium antibodies, $t T G$ anti-tissue transglutaminase antibodies

${ }^{*}$ Few patients had more than one positive CD markers

frequency and distribution of serologic markers of CD.

All patients were asymptomatic, but one JIA patient with positive tTG had recurrent abdominal pain and chronic diarrhea. None of the patients with isolated AGAA or AGAG antibodies had gastrointestinal symptoms. All patients with positive serologic markers of CD were offered duodenal-jejunal endoscopic biopsy, including 17 patients with positive EmA and tTG and markedly elevated AGAA and AGAG and 10 patients with isolated and slightly elevated AGAA and AGAG levels. Two patients declined the procedure, while the rest of the patients underwent endoscopic biopsy. All aSLE and cSLE patients had normal mucosa, but one RA and one JIA patient with positive EmA and tTG showed typical histologic findings of CD and were therefore diagnosed with $\mathrm{CD}$. None of the patients with isolated slightly elevated AGAA and AGAG were diagnosed with CD.

Most of the aSLE and cSLE patients demonstrated active disease with a SLEDAI mean of 9.0 (9.7). Patients with positive CD markers were comparable to those with negative markers. No association was observed between the positivity of CD markers and SLE activity. Furthermore, positivity of CD markers was not correlated with SLE-related antibodies (ANA, ds-DNA, APL) or complement levels. HLA-DQ2 and -DQ8 testing was requested for two aSLE patients with positive tTG markers. One patient with normal biopsy tested positive for HLA-DQ2, while the second patient who declined the endoscopic biopsy tested negative for HLA-DQ2 and HLADQ8.

\section{DISCUSSION}

Population-based screening studies have shown a high incidence of CD among adults and children in Saudi Arabia [10, 27], and there is wide heterogeneity in the CD presentation. However, most affected individuals have gastrointestinal manifestations, including chronic diarrhea and malabsorption, which result in weight loss and can lead to growth and developmental retardation. Unfortunately, CD remains unrecognized as recent insights have shown that symptoms of CD may also be silent or subclinical. Definite diagnosis of $\mathrm{CD}$ requires clinical symptoms, positive serologic test results, and histologic findings along with improvement in clinical symptoms upon initiating a gluten-free diet. Thus, screening tests, particularly in high-risk patients, might expedite the diagnostic process and help to decide if further histologic biopsy is 
needed [28-30]. The occurrence of CD was high among autoimmune disease patients. The prevalence of CD varies among SLE patient groups; several reports showed a greater coexistence of CD among patients with SLE than among their matched controls [29, 31]. There is similarity between the pathogenesis of CD and SLE, as both share a common genetic predilection favored by the presence of HLAB8 and HLA DR3 [15]. However, the exact association between SLE and CD is still unknown. Although SLE and CD have a higher correlation in their immune system response and incorporate a complex prototype that has an enormous effect on other systems and metabolic functions, this resulting complexity displays numerous variables that make it difficult to identify the actual condition. Consequently, if serologic CD markers are not tested at any stage, there is a possibility of incorrect diagnosis and management, which can lead to unnecessary exposure to immunosuppressive medications, with their related side effects. Autoantibodies to organspecific autoimmune diseases comprising CD markers are found in patients with SLE. However, several studies have shown variations in the positivity of CD markers, which could be elucidated by alterations in the screening method and studied population or concomitant immunosuppressive medication [31-34]. Although the frequency of CD markers is considered to be high in SLE patients, only EmA and tTG showed significant differences with respect to controls [34, 35].

Our study showed an increased proportion of adults and children with SLE testing positive for at least one serologic CD marker, but a comparable proportion to the rate of $\mathrm{CD}$ marker positivity among patients with RA and JIA. Of the studied SLE patients, 9.6\% tested positive for anti-gliadin antibodies (either AGAG or AGAA), while tTG was found in $3.5 \%$ and $\mathrm{EmA}$ was positive in $2.6 \%$. Additionally, few patients were positive for multiple serologic CD markers, but this had no impact on the clinical findings. There was no correlation between SLE disease activity and other autoantibodies. Patients with SLE had more frequent weak AGAG and AGAA positivity, while arthritis patients had more positive EmA and tTG, but the difference was not significant.

Interestingly, in our series of SLE patients that tested positive for CD markers, none had clinical symptoms suggesting $\mathrm{CD}$, and histologic examination of small bowel biopsy did not confirm CD. Our observations are consistent with previously published reports [34, 36]. These findings may be explained by non-specific polyclonal activation of B cells and cross-reactivity with other autoantibodies. Additionally, it is probable that the use of immunosuppressive medications may alter the histologic findings. Few case reports of autoimmune diseases and CD treated with corticosteroids and immunosuppressive medications have highlighted the potential consequences of these medications on enteropathy [32, 33]. Serologic CD markers are widely used as a screening tool for CD diagnosis. However, our results, similar to those of previous studies, questioned whether the isolated serologic $C D$ marker positivity, particularly AGAG or AGAA, is sufficient evidence to proceed to endoscopic and histologic examination [33].

To the best of our knowledge, this is the first study in the Middle East that highlights the importance of determining CD among SLE patients. The limitations of this study include the lack of healthy controls, small number of patients, single visit assessment, and solitary center involvement; further studies are required to evaluate the relationship between SLE and $\mathrm{CD}$ and its impact on disease activity and severity.

\section{CONCLUSION}

This study confirms an increased frequency of positive serologic $\mathrm{CD}$ markers in patients with SLE. However, the accuracy of these markers, particularly AGAG or AGAA, in asymptomatic SLE patients remains unconfirmed. Furthermore, the presence of serologic CD markers had no impact on clinical presentation and comorbidity and did not influence SLE disease activity. 


\section{ACKNOWLEDGEMENTS}

The authors thank the patients, investigators, and investigative staff who contributed to 'The value of screening for celiac disease in systemic lupus erythematosus'

Funding. No funding or sponsorship was received for this study or publication of this article. The Rapid Service Fee was funded by the authors.

Authorship. All named authors meet the International Committee of Medical Journal Editors criteria for authorship for this article, take responsibility for the integrity of the work as a whole, and have given their approval for this version to be published.

Authorship Contributions. All authors were involved in drafting the manuscript and reviewing and revising it critically for important intellectual content. Sulaiman M Al-Mayouf, Fahidah AlEnzi, and AlShaikh Ahmed contributed to the conception and study design, acquisition, analysis, and interpretation of data. Fahidah AlEnzi, Mada Yateem, and Badryah Alhaymouni contributed to the data collection. Manal Shaikh contributed to the analysis and interpretation of data. Fahad AlSohaibani contributed to endoscopy and analysis of celiac antibodies. All authors read and approved the final version.

Prior Presentation. This manuscript is based on work that was previously presented as a poster at the 20th Asia Pacific League of Associations for Rheumatology Congress (APLAR), 6-9 September 2018, Kaohsiung, Taiwan, and the abstract was published in a special issue of the International Journal of Rheumatic Diseases (DOI: 10.1111/1756-185X.13361).

Disclosures. Fahidah AlEnzi, Mada Yateem, Manal Shaikh, Fahad AlSohaibani, Badryah Alhaymouni, AlShaikh Ahmed, and Sulaiman M Al-Mayouf have nothing to disclose.

Compliance with Ethics Guidelines. The study was approved by the Research Affairs
Council at KFSHRC-R. All collected data were analyzed with due confidentiality practice, and no personal identity was needed. All procedures performed involving human participants were performed in accordance with the ethical standards of the institutional and/or national research committee and within the 1964 Helsinki Declaration and its later amendments or comparable ethical standards. Informed consent was obtained from all adult participants and from the parents of all enrolled children.

Data Availability. The datasets generated during and/or analyzed during the current study are available from the corresponding author on reasonable request.

Open Access. This article is licensed under a Creative Commons Attribution-NonCommercial 4.0 International License, which permits any non-commercial use, sharing, adaptation, distribution and reproduction in any medium or format, as long as you give appropriate credit to the original author(s) and the source, provide a link to the Creative Commons licence, and indicate if changes were made. The images or other third party material in this article are included in the article's Creative Commons licence, unless indicated otherwise in a credit line to the material. If material is not included in the article's Creative Commons licence and your intended use is not permitted by statutory regulation or exceeds the permitted use, you will need to obtain permission directly from the copyright holder. To view a copy of this licence, visit http://creativecommons.org/licenses/by$\mathrm{nc} / 4.0 /$.

\section{REFERENCES}

1. Fawzy M, Edrees A, Okasha H, El Ashmaui A, Ragab G. Gastrointestinal manifestations in systemic lupus erythematosus. Lupus. 2016;25(13):1456-62.

2. Brewer BN, Kamen DL. Gastrointestinal and hepatic disease in systemic lupus erythematosus. Rheum Dis Clin N Am. 2018;44(1):165-75. 
3. Mader R, Adawi M, Schonfeld S. Malabsorption in systemic lupus erythematosus. Clin Exp Rheumatol. $1997 ; 15(6): 659-61$.

4. Sherman Y, Karanicolas R, DiMarco B, Pan N, Adams AB, Barinstein LV, et al. Unrecognized celiac disease in children presenting for rheumatology evaluation. Pediatrics. 2015;136(1):e68-75. https:// doi.org/10.1542/peds.2014-2379.

5. Jericho H, Assiri A, Guandalini S. Celiac disease and wheat intolerance syndrome: a critical update and reappraisal. J Pediatr Gastroenterol Nutr. 2017;64(1):15-211.

6. Gujral N, Freeman HJ, Thomson AB. Celiac disease: prevalence, diagnosis, pathogenesis and treatment. World J Gastroenterol. 2012;18(42):6036-59.

7. Laurikka P, Nurminen S, Kivelä L, Kurppa K. Extraintestinal manifestations of celiac disease: early detection for better long-term outcomes. Nutrients. 2018;10(8):1015. https://doi.org/10. 3390/nu10081015.

8. Singh P, Arora A, Strand TA, Leffler DA, Catassi C, Green $\mathrm{PH}$, et al. Global prevalence of celiac disease: systematic review and meta-analysis. Clin Gastroenterol Hepatol. 2018;16(6):823-36.

9. Grode L, Bech BH, Jensen TM, Humaidan P, Agerholm IE, Plana-Ripoll $O$, et al. Prevalence, incidence, and autoimmune comorbidities of celiac disease: a nation-wide, population-based study in Denmark from 1977 to 2016. Eur J Gastroenterol Hepatol. 2018;30(1):83-91.

10. Al-Hussaini A, Troncone R, Khormi M, AlTuraiki M, Alkhamis W, Alrajhi $M$, et al. Mass screening for celiac disease among school-aged children: toward exploring celiac iceberg in Saudi Arabia. J Pediatr Gastroenterol Nutr. 2017;65(6):646-51.

11. Roy A, Laszkowska M, Sundström J, Lebwohl B, Green PH, Kämpe $\mathrm{O}$, et al. Prevalence of celiac disease in patients with autoimmune thyroid disease: a meta-analysis. Thyroid. 2016;26(7):880-90.

12. Conti V, Leone MC, Casato M, Nicoli M, Granata G, Carlesimo M. High prevalence of gluten sensitivity in a cohort of patients with undifferentiated connective tissue disease. Eur Ann Allergy Clin Immunol. 2015;47(2):54-7.

13. Robazzi T, Adan L, Pimentel K, Guimarães I, Magalhães J, Toralles $\mathrm{M}$, et al. Autoimmune endocrine disorders and coeliac disease in children and adolescents with juvenile idiopathic arthritis and rheumatic fever. Clin Exp Rheumatol. 2013;31(2): 310-7.
14. Al-Mayouf SM, Al-Mehaidib AI, Alkaff MA. The significance of elevated serologic markers of celiac disease in children with juvenile rheumatoid arthritis. Saudi J Gastroenterol. 2003;9(2):75-8.

15. Zhernakova A, Withoff S, Wijmenga C. Clinical implications of shared genetics and pathogenesis in autoimmune diseases. Nat Rev Endocrinol. 2013;9(11):646-59.

16. Ludvigsson JF, Rubio-Tapia A, Chowdhary V, Murray JA, Simard JF. Increased risk of systemic lupus erythematosus in 29,000 patients with biopsy-verified celiac disease. J Rheumatol. 2012;39(10): 1964-70.

17. Forbess LJ, Gordon JK, Doobay K, Bosworth BP, Lyman S, Davids ML, et al. Low prevalence of coeliac disease in patients with systemic sclerosis: a cross-sectional study of a registry cohort. Rheumatology. 2013;52(5):939-43.

18. Vives M-J, Esteve M, Marine M, Fernendez-Baeares F, Alsina M, Salas A, et al. Prevalence and clinical relevance of enteropathy associated with systemic autoimmune diseases. Dig Liver Dis. 2012;44(8): 636-42.

19. Petri M, Orbai AM, Alarcon GS, Gordon C, Merrill JT, Fortin PR, et al. Derivation and validation of the systemic lupus international collaborating clinics classification criteria for systemic lupus erythematosus. Arthritis Rheum. 2012;64(8):2677-86.

20. Sokolove J, Strand V. Rheumatoid arthritis classification criteria. Bull NYU Hosp Jt Dis. 2010;68(3): 232-8.

21. Petty RE, Southwood TR, Manners P, Baum J, Glass $\mathrm{DN}$, Goldenberg $\mathrm{J}$, et al. International League of Associations for Rheumatology classification of juvenile idiopathic arthritis: second revision, Edmonton, 2001. J Rheumatol. 2004;31(2):390-2.

22. Gutierrez Suerez R, Ruperto N, Gastaldi R, Pistorio A, Felici E, Vargas RB, et al. A proposal for a pediatric version of the Systemic Lupus International Collaborating Clinics/American College of Rheumatology Damage Index based on the analysis of 1,015 patients with juvenile onset systemic lupus erythematosus. Arthritis Rheum. 2006;54(9): 2989-96.

23. Armstrong D, Don-Wauchope AC, Verdu EF. Testing for gluten-related disorders in clinical practice: the role of serology in managing the spectrum of gluten sensitivity. Can J Gastroenterol. 2011;25(4): 193-7. https://doi.org/10.1155/2011/642452.

24. Salas-Cuestas F, Bautista-Molano W, Bello-Gualtero JM, Arias I, Castillo DM, Chila-Moreno L, et al. Higher levels of secretory $\operatorname{IgA}$ are associated with 
low disease activity index in patients with reactive arthritis and undifferentiated spondyloarthritis. Front Immunol. 2017. https://doi.org/10.3389/ fimmu.2017.00476.

25. Marginean CO, Meliț LE, Mareş R-C, Mărginean MO, Voidăzan S, Dobreanu M. Clinical and biological correlations in celiac disease in children: the prospective single experience of a romanian tertiary center: a case-control study (Strobe-Compliant study). Medicine. 2017;96(20):e6936. https://doi. org/10.1097/MD.0000000000006936.

26. Almutairi N, Aljaser A, Almutairi A, Alshaikh M, Eldali A, Al-Mayouf SM. Utility of serum ferritin and soluble interleukin-2 receptor as markers of disease activity in childhood systemic lupus erythematosus. Int J Pediatr Adolesc Med. 2019. https://doi. org/10.1016/j.ijpam.2019.07.007.

27. Aljebreen AM, Almadi MA, Alhammad A, Al Faleh FZ. Seroprevalence of celiac disease among healthy adolescents in Saudi Arabia. World J Gastroenterol. 2013;19(15):2374-8.

28. White LE, Bannerman E, Gillett PM. Coeliac disease and the gluten free diet: a review of the burdens; factors associated with adherence and impact on health related quality of life, with specific focus on adolescence. J Hum Nutr Diet. 2016;29(5):593-606.

29. Chou R, Bougatsos C, Blazina I, Mackey K, Grusing S, Selph S. Screening for celiac disease: evidence report and systematic review for the US Preventive Services Task Force. JAMA. 2017;317(12):1258-68.

30. Sahin Y, Evliyaoglu O, Erkan T, Cokugras FC, Ercan $\mathrm{O}$, Kutlu $\mathrm{T}$. The frequency of celiac disease in children with autoimmune thyroiditis. Acta Gastroenterol Belg. 2018;81(1):5-8.

31. Dahan S, Shor DB, Comaneshter D, Tekes-Manova $\mathrm{D}$, Shovman $\mathrm{O}$, Amital $\mathrm{H}$, et al. All disease begins in the gut: celiac disease co-existence with SLE. Autoimmun Rev. 2016;15(8):848-53.

32. Iqbal U, Chaudhary A, Karim MA, Anwar H, Merrella N. Refractory celiac disease successfully treated with azathioprine. Gastroenterology Res. 2017;10(3):199-201. https://doi.org/10.14740/ gr819w.

33. Koehne V, Bahia M, Lanna C, Pinto M, Bambirra E, Cunha A. Prevalence of serological markers for celiac disease (IgA and IgG class antigliadin antibodies and IgA class antiendomysium antibodies) in patients with autoimmune rheumatologic diseases in Belo Horizonte, MG Brazil. Arq Gastroenterol. 2010;47(3):250-6.

34. Picceli VF, Skare TL, Nisihara R, Kotze L, MessiasReason I, Utiyama SRR. Spectrum of autoantibodies for gastrointestinal autoimmune diseases in systemic lupus erythematosus patients. Lupus. 2013;22(11):1150-5.

35. Caio G, De Giorgio R, Ursini F, Fanaro S, Volta U. Prevalence of celiac disease serological markers in a cohort of Italian rheumatological patients. Gastroenterol Hepatol Bed Bench. 2018;11(3):244-9.

36. Shaffer T, Fink S, Kopecky C, Grissmer L, Enzenhauer R, Kadakia S, et al. The prevalence of celiac disease autoantibodies in patients with systemic lupus erythematosus. Am J Gastroenterol. 2001;96(4):1113-5. https://doi.org/10.1016/S00029270(01)02316-4. 